\title{
INTERACTION OF SHOCK WAVE WITH MULTI-FLUIDS INTERF ACE USING QUADRILATERAL-BASED ADAPTIVE MESH
}

\author{
H. W. ZHENG*, X. J. LI and G. W. YANG \\ LHD, Institute of Mechanics, Chinese Academy of Sciences \\ 15 Beisihuanxi Road, Beijing 100190, P. R. China \\ *h.zheng@imech.ac.cn \\ C. SHU \\ Department of Mechanical Engineering \\ National University of Singapore \\ 10 Kent Ridge Crescent 119260, Singapore \\ mpeshuc@nus.edu.sg \\ Received 9 November 2011 \\ Accepted 20 March 2012 \\ Published 9 May 2012
}

\begin{abstract}
In this paper, the interaction of shock waves with multi-fluids interfaces is investigated by numerical simulations using unstructured quadrilateral adaptive meshes. In order to obtain a detailed structure of the interface, a solution adaptive method for compressible multi-fluid flows developed by Zheng et al. is employed. Firstly, the method is verified by a planar shock and interface interaction problem, which is compared with the front tracking method for the Richtmyer-Meshkov instability problem. Following the verification, the interaction between a circular shock and a sinusoidally perturbed circular interface in cylinder vessel is firstly investigated in our paper. The results show that the solution adaptive method can be employed to study the compressible multi-fluid cases with relatively complex geometry as well as capturing the fine details of interfacial structures of the interaction.
\end{abstract}

Keywords: Shock; multi-fluids; interface; unstructured; adaptive mesh refinement; quadrilateral.

PACS Nos.: 11.25.Hf, 123.1K.

\section{Introduction}

The instability induced by shock and interface interaction is a fundamental instability in both nature, such as supernova explosions in astrophysics, ${ }^{1-4}$ and industrial applications such as inertial confinement fusion (ICF) capsule design. ${ }^{5}$ It has been extensively studied experimentally, ${ }^{6,7}$ numerically, ${ }^{8-12}$ and analytically. ${ }^{13}$

Most previous numerical works ${ }^{8-12}$ can be grouped into two types. One is the instability by impulsive acceleration of a planar shock on slightly perturbed 
interfaces. ${ }^{9,10}$ Another is the instability induced by the interaction between a circular shock and a perturbed circular interface. ${ }^{11,12}$ The present work is intended to study the circular interfaces interaction with circular shocks with a non-Cartesian more feature-aligned quadrilateral mesh.

In order to gain a reliable description of the evolution of the small-scale structures, the adaptive technique is a suitable tool to be employed. During the past years, little work can be done in using adaptive method to study the shock interface interaction. To our knowledge, only the level set method on adaptive Cartesian grid ${ }^{10}$ and the level set method on overlapping adaptive Cartesian grid $^{14}$ have been used to simulate the planar shock and interface interaction problem. The main problem is the adaptive Cartesian grid is difficult to be applied to solving the problems with complex interfaces. Thus, in order to investigate the circular shocks and interface interaction problem, we adopt the recently developed adaptive method for multi-fluid flows (Zheng et $a .^{15}$ ) in this paper.

The rest of the paper is organized as follows. In the second section, the solution adaptive numerical method for compressible multi-fluid flows is presented. The numerical results are given in the third section. The model is firstly validated by the instability induced by the interaction between an initially perturbed interface and a planar shock. It will be applied to study the interaction between a circular shock and an initially perturbed circular interface. All these results show that the solution adaptive method can be used to produce the high resolution interfacial structure for the shock interface instability problem.

\section{Governing Equations}

In this section, the numerical method for the compressible multi-fluid flows with stiffened gas equation of state (EOS) is described. To capture the interface accurately, we also adopt the quadrilateral cell based unstructured adaptive method which is recently proposed by Zheng et al. ${ }^{15}$ To tackle the well-known difficulty ${ }^{9,16}$ of spurious pressure oscillations at material interfaces, the governing equations for the compressible multi-fluid flows are chosen as

$$
\partial_{t} \mathbf{U}+\nabla \cdot \mathbf{F}=\mathbf{S}
$$

where the vector of conservative variables, flux vector, and the source terms are

$$
\mathbf{U}=\left(\begin{array}{c}
\rho \\
\rho \mathbf{u} \\
E \\
\beta \\
\theta
\end{array}\right), \quad \mathbf{F}=\left(\begin{array}{c}
\rho \mathbf{u} \\
\rho \mathbf{u} \otimes \mathbf{u}+p[I] \\
(E+p) \mathbf{u} \\
\beta \mathbf{u} \\
\theta \mathbf{u}
\end{array}\right), \quad \mathbf{S}=\left(\begin{array}{c}
0 \\
0 \\
0 \\
\beta \nabla \cdot \mathbf{u} \\
\theta \nabla \cdot \mathbf{u}
\end{array}\right)
$$

where $\rho$ is the density, $\mathbf{u}$ is the velocity, and $E$ is the total energy. $\beta$ and $\theta$ are functions of properties of the material $(\gamma$ and $\pi) . \beta$ is also used to indicate the 
position of the interface. They are defined as

$$
\beta=\frac{1}{\gamma-1}, \quad \theta=\frac{\gamma \pi}{\gamma-1} .
$$

These two variables are also used to calculate the pressure $p$ according to stiffened gas EOS,

$$
p=\left(E-\frac{1}{2} \rho u^{2}+\theta\right) / \beta
$$

\section{Solution Adaptive Method}

In this section, the time evolution for the unstructured quadrilateral mesh-based adaptive solver ${ }^{15}$ is described. The data structure of the adaptive mesh ${ }^{15}$ consists of nodes, faces and cells. All nodes, faces and cells are stored in different dynamically allocated lists which are well-designed that the adding, deleting of a certain object is only of the order of $\mathrm{O}(1)$. After each refinement of a cell, each cell is split into four cells and each edge of this cell is split into two sub-edges (Fig. 1). These cells are further organized into different lists according to their level [Fig. 1(a)] and the edges are separated further stored into two lists: LeafEdgeList for leaf edges and

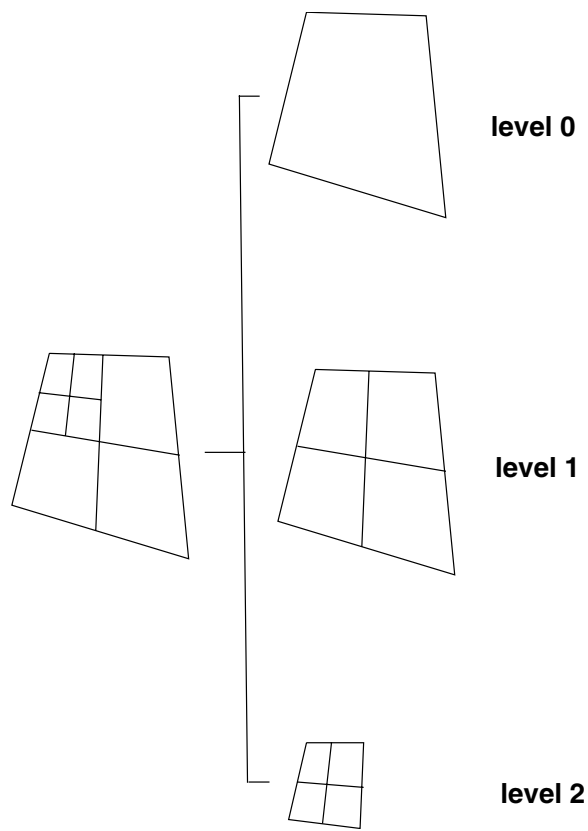

(a)

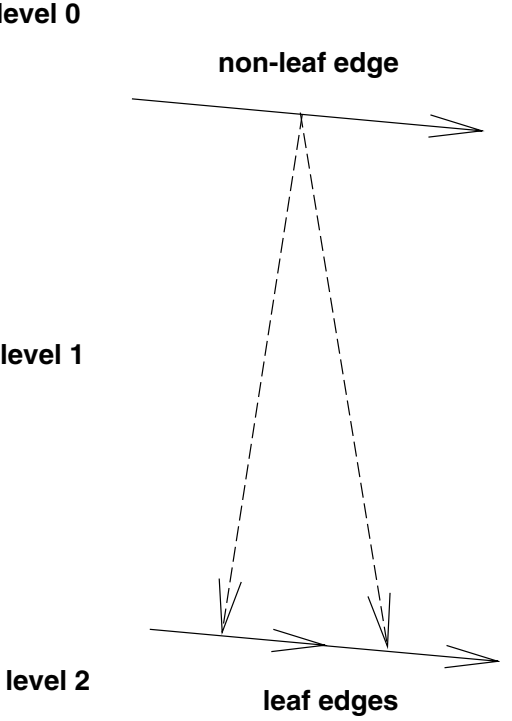

(b)

Fig. 1. Mesh. 
MotherEdgeList for non-leaf edges [Fig. 1(b)]. Due to the separated storage of leaf edges and non-leaf edges, the edge-based finite volume solver can be easily applied.

The set of Eq. (1) can be easily discretized at each leaf cell by the two stage Runge-Kutta schemes, ${ }^{15}$

$$
\mathbf{U}_{c}^{(*)}=\mathbf{U}_{c}^{n}-\alpha \operatorname{Res}\left(U_{c}^{n}\right)
$$

and

$$
\mathbf{U}_{c}^{n+1}=0.5\left[\mathbf{U}_{c}^{n}+\mathbf{U}_{c}^{(*)}-\alpha \mathbf{R}_{c}\left(\mathbf{U}_{c}^{(*)}\right)\right]
$$

where $\Delta t$ is the time step, $A_{c}$ is the area of cell $c, \mathbf{R}_{c}$ is the residual, and $\alpha$ is the ratio between time step and area $\left(\alpha=\Delta t / A_{c}\right)$.

The edge-based technique is employed to calculate the residual. That is, the residual $\left(\mathbf{R}_{f 1 \rightarrow L}\right)$ at the left neighboring cell center $(f 1 \rightarrow L$ or cell $\mathrm{C}$ in Fig. 2$)$ of a leaf edge $f 1$ is updated in the following way,

$$
\mathbf{R}_{f 1 \rightarrow L}=\operatorname{Res}_{f 1 \rightarrow L}+\Phi_{f 1}\left(\mathbf{U}^{-}, \mathbf{U}^{+}, \mathbf{n}_{f 1}\right) \cdot \Delta l_{f 1}
$$

and the residual $\left(\mathbf{R}_{f 1 \rightarrow R}\right)$ at the right cell center $(f 1 \rightarrow R$ or cell $\mathrm{E}$ in Fig. 2$)$ of this leaf edge $f 1$ is updated by

$$
\mathbf{R}_{f 1 \rightarrow R}=\mathbf{R}_{f 1 \rightarrow R}-\Phi_{f 1}\left(\mathbf{U}^{-}, \mathbf{U}^{+}, \mathbf{n}_{f 1}\right) \cdot \Delta l_{f 1} .
$$

To discretize the convection term, the Harten, Lax and van Leer approximate Riemann solver with the Contact wave restored (HLLC) numerical flux for an edge is adopted for compressible multi-fluid flows, ${ }^{15}$

$$
\Phi_{f}\left(U^{-}, U^{+}, \mathbf{n}\right)=\frac{1}{2}\left[\Phi^{-}+\Phi^{+}-\operatorname{sign}\left(s_{m}\right)\left(\mathbf{U}^{+}-\mathbf{U}^{-}\right)\right]
$$

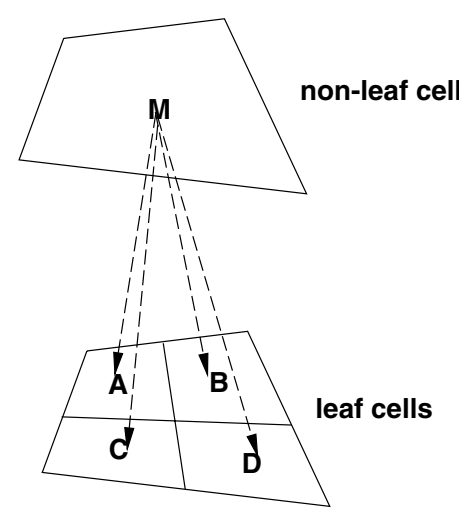

(a)

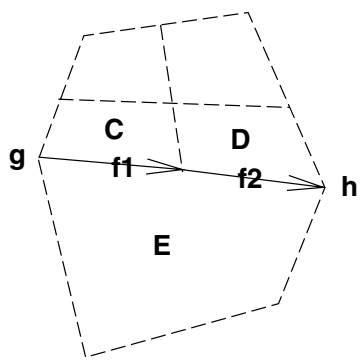

(b)

Fig. 2. Evolution on leaf cells. 
with

$$
\Phi^{j}=\mathbf{F}\left(\mathbf{U}^{j}\right) \cdot \mathbf{n}+s_{j}^{\#}\left(\mathbf{U}^{* j}-\mathbf{U}^{j}\right), \quad j=-,+,
$$

where the intermediate states can be calculated by

$$
\mathbf{U}^{* j}=\frac{s_{j}-u_{n}^{j}}{s_{j}-s_{m}}\left(\begin{array}{c}
\rho^{j} \\
\rho^{j}\left[u_{1}^{j}-\left(u_{n}^{j}-s_{m}\right) n_{x}\right] \\
\rho^{j}\left[u_{2}^{j}-\left(u_{n}^{j}-s_{m}\right) n_{y}\right] \\
E^{j} \\
\beta^{j} \\
\theta^{j}
\end{array}\right)+\left(\begin{array}{c}
0 \\
0 \\
0 \\
\quad j=-,+
\end{array}\right)
$$

with

$$
p^{* j}=\rho^{j}\left(u_{n}^{j}-s_{j}\right)\left(u_{n}^{j}-s_{m}\right)+p^{j}, \quad j=-,+.
$$

Here, $s_{j}$ and $p^{* j}$ are the intermediate signal speeds and pressures of HLLC scheme. ${ }^{15}$

In order to achieve the object of the spurious pressure oscillations-free material interfaces under complex geometry, the solving of the last two sub-equations in Eq. (1) are important. In this paper, we adopt a HLLC consistent way in paper, ${ }^{15}$ the HLLC scheme is embedded by updating the last two items of residue as

$$
\begin{aligned}
& \mathbf{R}_{c, 4}=\mathbf{R}_{c, 4}-\beta_{c} \sum_{f} q_{f} \cdot \Delta l_{f}, \\
& \mathbf{R}_{c, 5}=\mathbf{R}_{c, 5}-\theta_{c} \sum_{f} q_{f} \cdot \Delta l_{f} .
\end{aligned}
$$

Here, $q_{f}$ is the HLLC intermediate normal velocity ${ }^{15}$ at the edge.

\section{Results and Discussion}

In order to investigate the instability problem induced by shock and interface interaction, several numerical tests in two dimensions are considered. The first case is an instability problem induced by a planar shock and an initially perturbed interface in a tube. It is used as a verification of the code. The second case is the instability problem which is triggered by the interaction between an initially perturbed circular interface and a circular shock inside circular geometry.

\subsection{Instability problem with planar air-SF6 interaction}

In this sub-section, a standard Richtmyer-Meshkov (RM) interface problem ${ }^{9}$ in a shock tube with length 4 and height 1 is considered. Initially, two fluids (air and SF6) are separated by a single mode perturbation interface (Fig. 3) that the initial location 


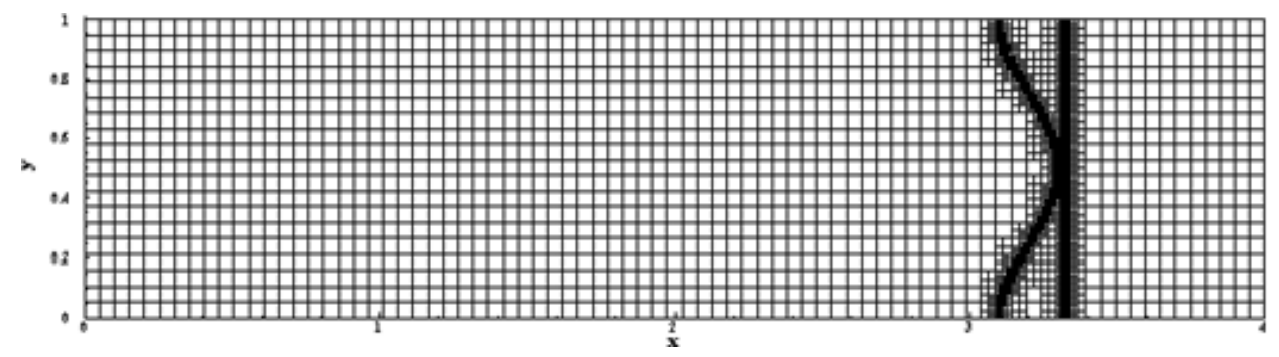

(a)

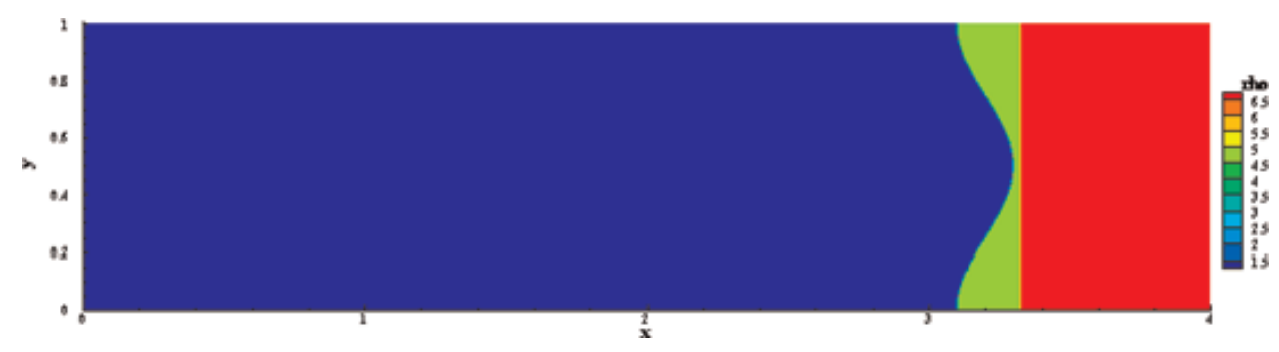

(b)

Fig. 3. (Color online) Mesh (a) and density contour (b) at time $t=0 \mathrm{~s}$.

of the interface is represented by

$$
x=x_{0}+a_{0} \cos [f(y-0.5)], \quad y \in[0,1],
$$

where $x_{0}$ is the location of the unperturbed interface, $a_{0}$ is the amplitude of the perturbation, and $f$ is the frequency.

To trigger the instability, a planar Mach 1.24 shock wave is put at in air propagating from the right (air) to the left (SF6) of the interface. These two un-shocked fluids are at rest with constant pressure $(1 \mathrm{~Pa})$ across the interface and the material properties are set as

$$
\rho_{\mathrm{SF} 6}=5.04 \mathrm{~kg} / \mathrm{m}^{3}, \quad \gamma_{\mathrm{SF} 6}=1.093, \quad \pi_{\mathrm{SF} 6}=0,
$$

for SF6, and

$$
\rho_{\text {air }}=1 \mathrm{~kg} / \mathrm{m}^{3}, \quad \gamma_{\text {air }}=1.4, \quad \pi_{\text {air }}=0,
$$

for air.

In terms of acoustic impedance (the density times the sound speed), SF6 is a heavy fluid and air is a light fluid. When the incident shock collides with the material interface, it bifurcates into a transmitted shock and a reflected wave (Fig. 4). Since the interface is accelerated by a shock wave coming from the light-fluid to the heavyfluid region, the reflected wave is a shock. After the colliding, the interface becomes unstable. At time $1 \mathrm{~s}$, the interface has begun to roll up (Fig. 5). It will finally develop to form the RM instability with the bubbles and spikes (Fig. 6). 
Interaction of Shock Wave with Multi-Fluids Interface using Quadrilateral-Based Adaptive Mesh

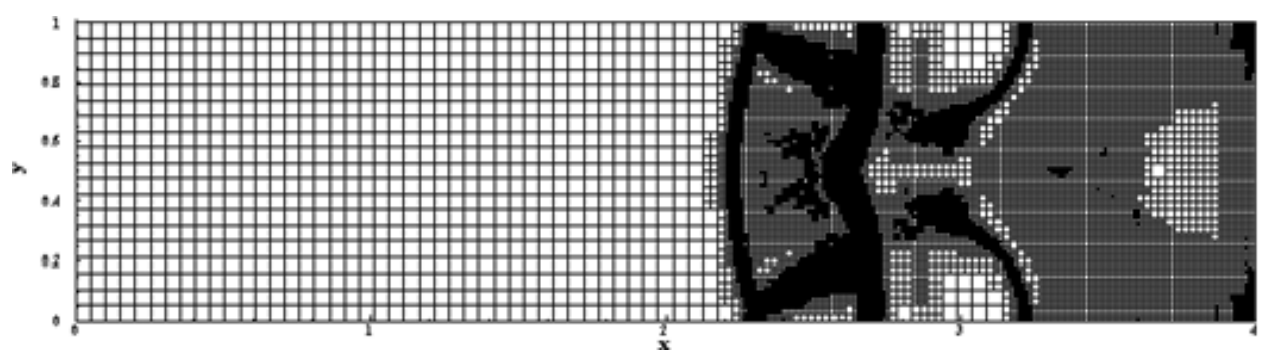

(a)

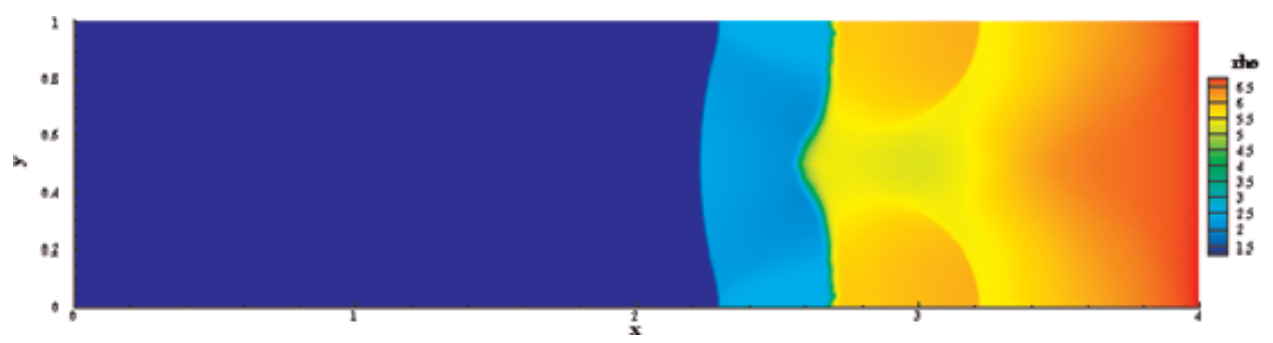

(b)

Fig. 4. (Color online) Mesh (a) and density contour (b) at time $t=0.5 \mathrm{~s}$.

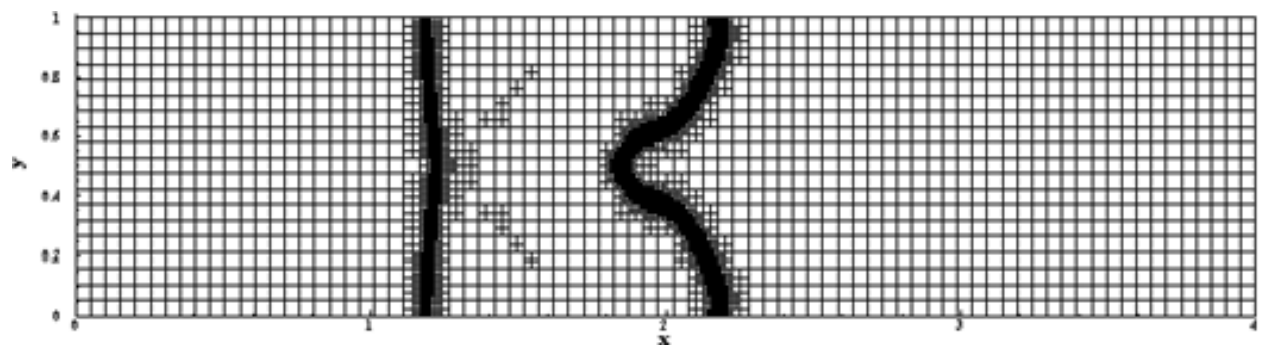

(a)

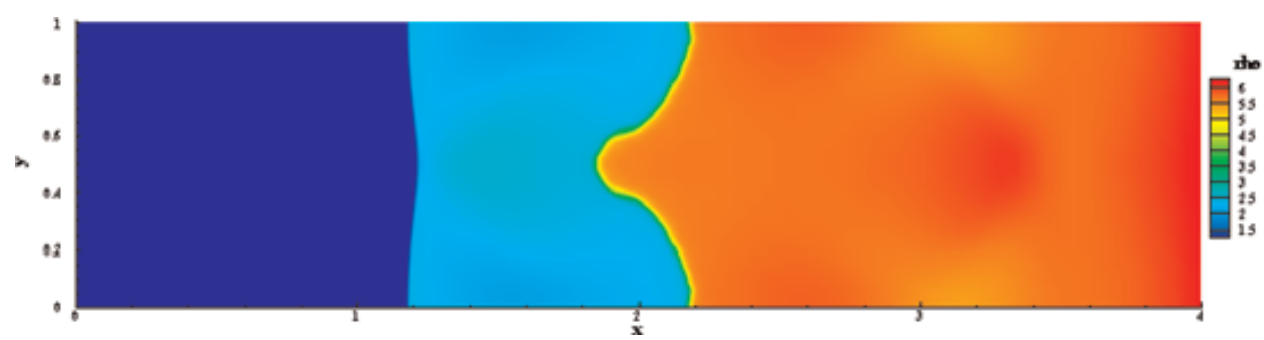

(b)

Fig. 5. (Color online) Mesh (a) and density contour (b) at time $t=1 \mathrm{~s}$. 


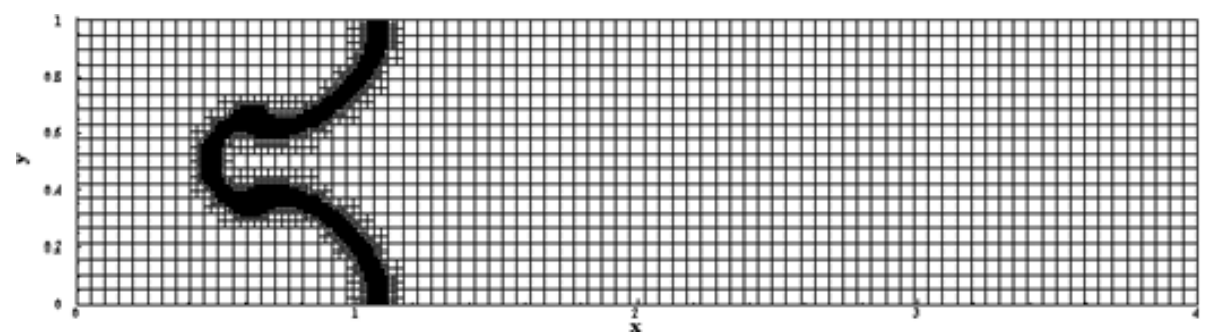

(a)

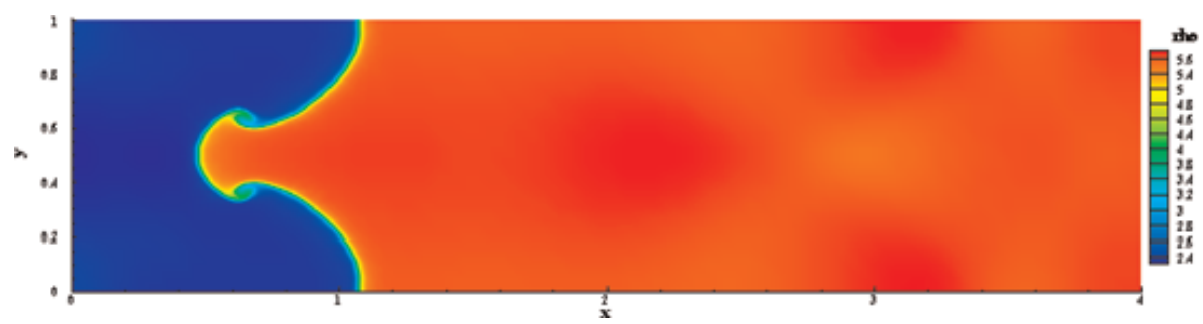

(b)

Fig. 6. (Color online) Mesh (a) and density contour (b) at time $t=2 \mathrm{~s}$.

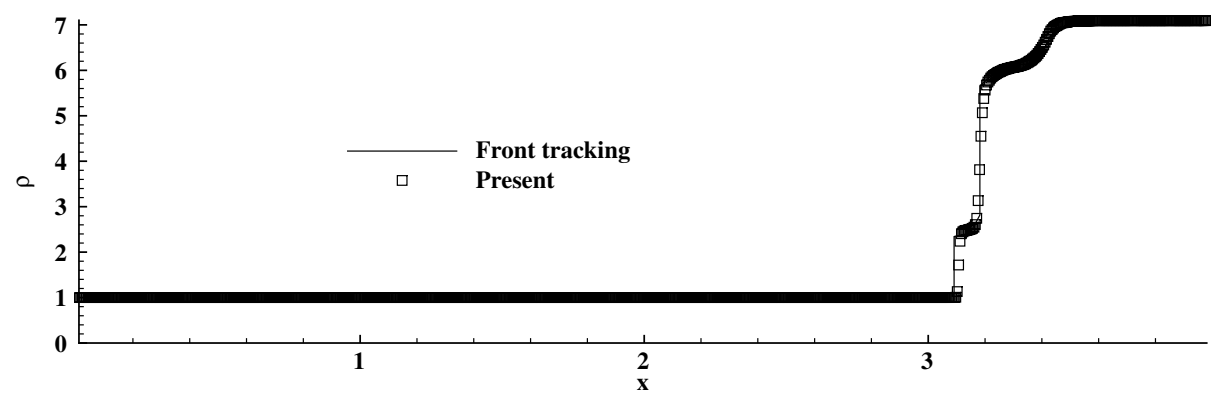

(a)

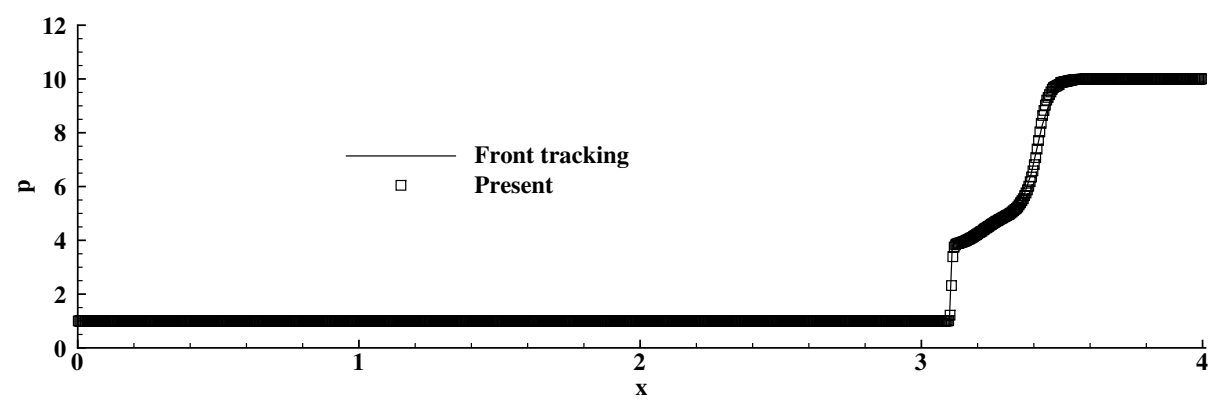

(b)

Fig. 7. Density (a) and pressure profile (b) at time $t=0.1 \mathrm{~s}$. 


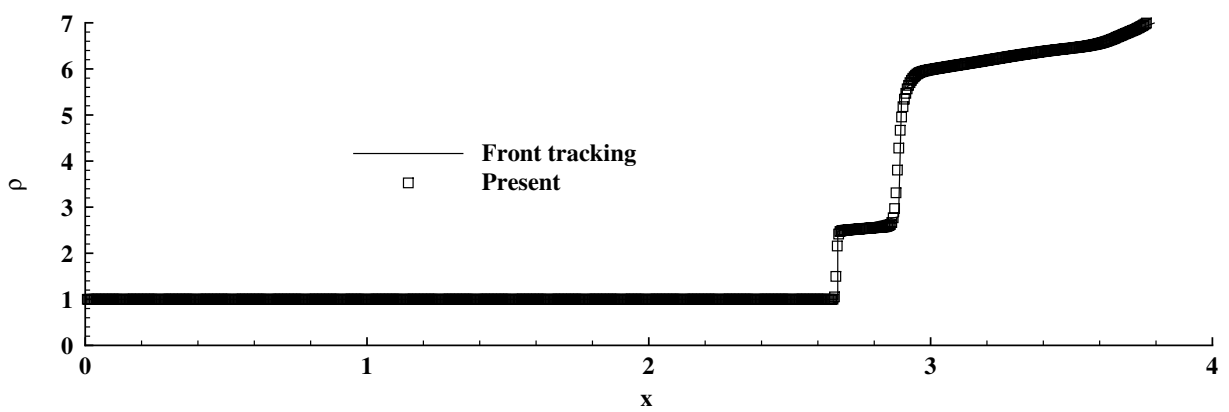

(a)

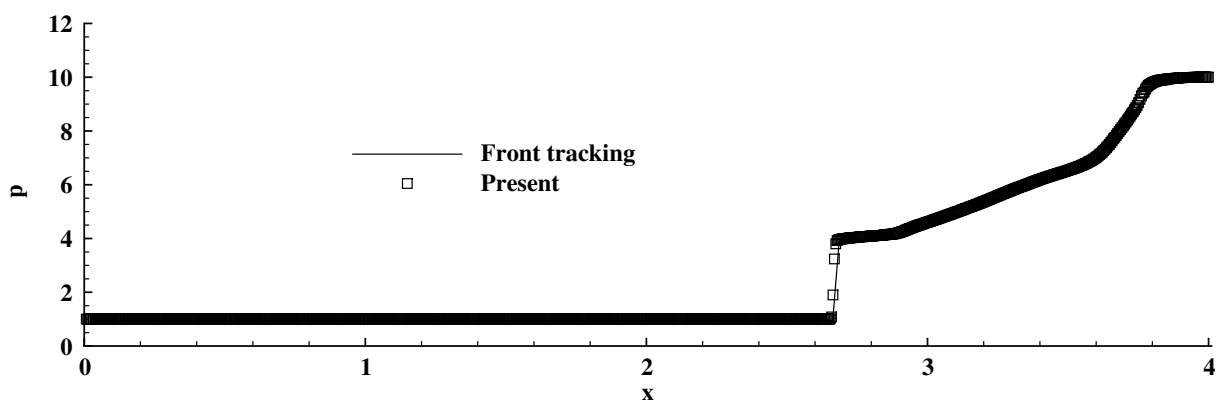

(b)

Fig. 8. Density (a) and pressure profile (b) at time $t=0.3 \mathrm{~s}$.

To evaluate the current code, the density profiles are plotted in Figs. 7-10. The present results are compared with the front tracking results ${ }^{9}$ which is obtained from the curves of Shyue ${ }^{9}$ by using the software of GetData Graph Digitizer 2.24. It is clear that our results agree well with those of Shyue ${ }^{9}$. This shows that our algorithm works well on the interfacial instability problem.

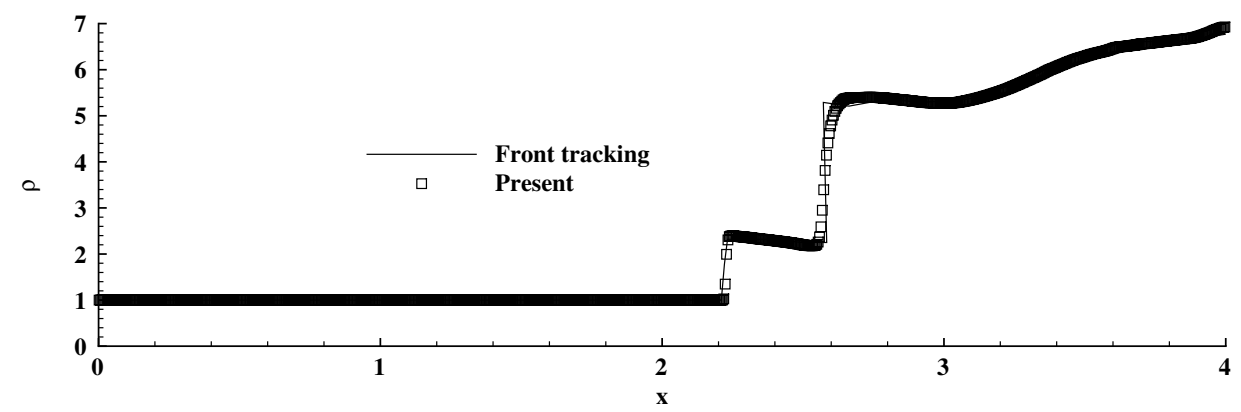

(a)

Fig. 9. Density (a) and pressure profile (b) at time $t=0.5 \mathrm{~s}$. 


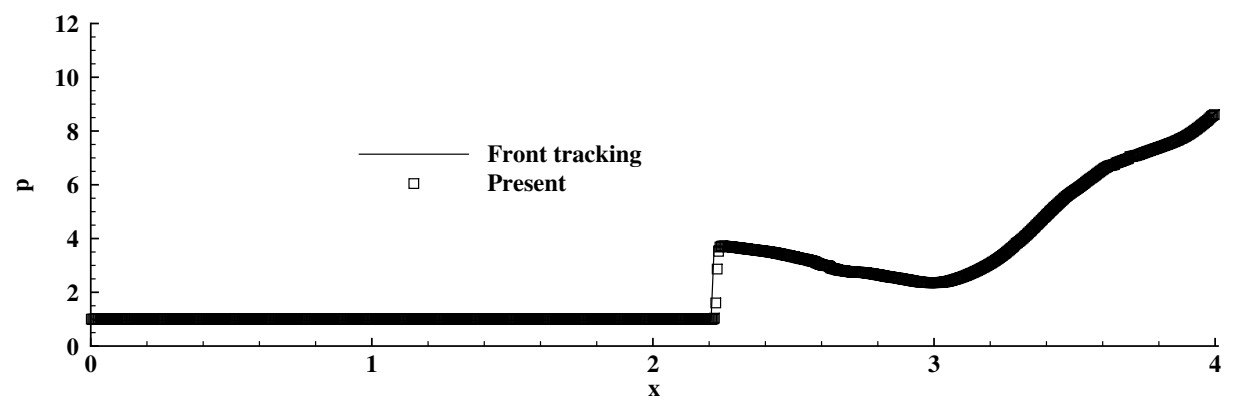

(b)

Fig. 9. (Continued)

\subsection{Interfacial instability by circular shock in cylindrical vessel}

We now investigate the shock interface interaction in a relatively complex geometry (cylindrical vessel with a radius as $12 \mathrm{~cm}$ ) on unstructured mesh as shown in Fig. 11. In contrast to the planar interface, the interface shape is a single-mode perturbation of a circle interface which is described by

$$
r=r_{0}+a_{0} \cos (f \phi) .
$$

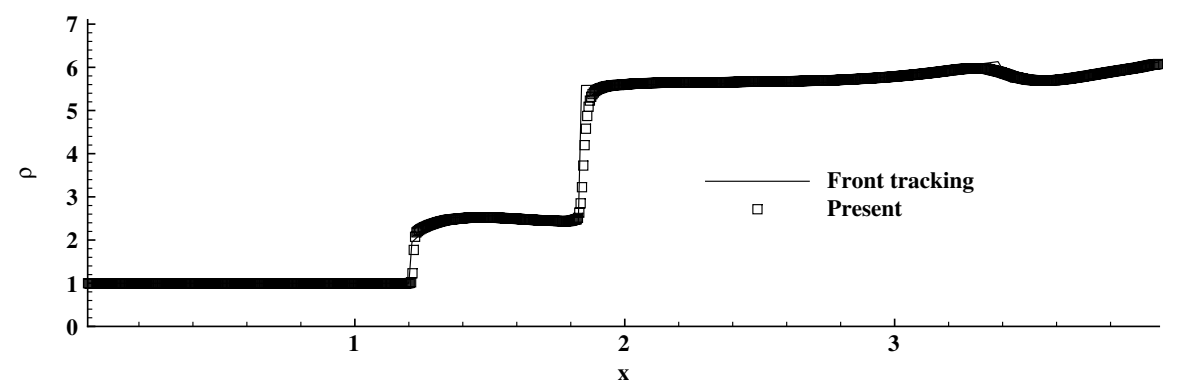

(a)

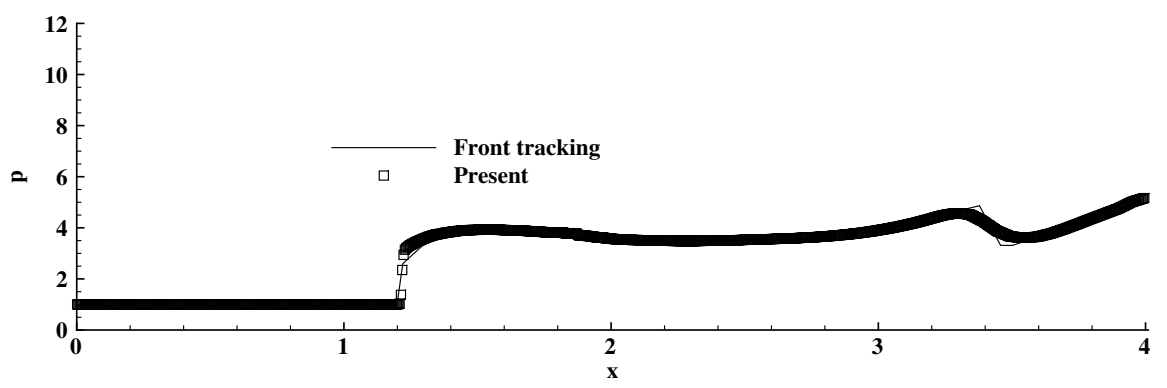

(b)

Fig. 10. Density (a) and pressure profile (b) at time $t=1 \mathrm{~s}$. 


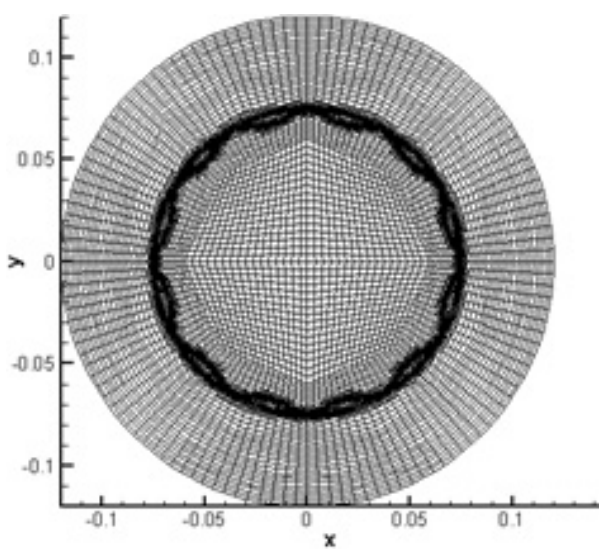

(a)

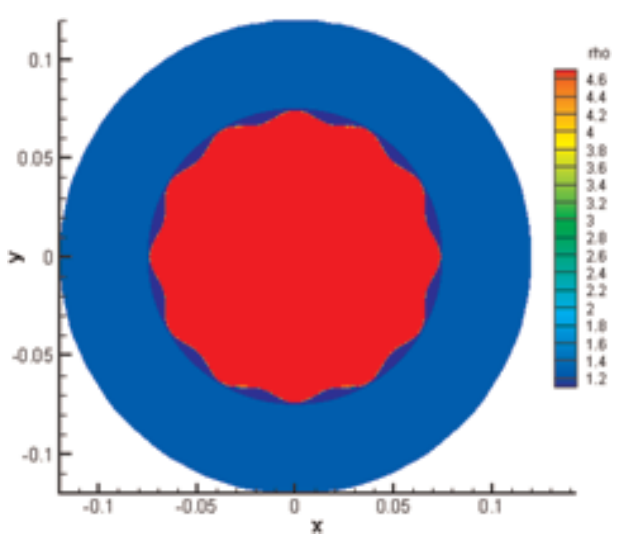

(b)

Fig. 11. (Color online) Mesh (a) and density contour (b) at time $t=0 \mathrm{~s}$.

Here, $r_{0}$ is the nominal radius, $\phi$ is the angle, and $a_{0}$ is the perturbation amplitude. The frequency $f$ is set to 12 and thus the wavelength is $3.75 \mathrm{~cm}$. The nominal radius $r_{0}$ is $7.162 \mathrm{~cm}$ and the perturbation amplitude $a_{0}$ is $0.24 \mathrm{~cm}$. Note that for this problem the wavelength changes dynamically as the interface moves inward.

This interface separates the two fluids as the two sections with the inner SF6 and outer air respectively. Initially, these un-shocked fluids are at rest with a uniform pressure $(80000 \mathrm{~Pa})$ across the interface and the material properties are set as

$$
\rho_{\text {air }}=0.95 \mathrm{~kg} / \mathrm{m}^{3}, \quad \gamma_{\text {air }}=1.4, \quad \pi_{\text {air }}=0
$$

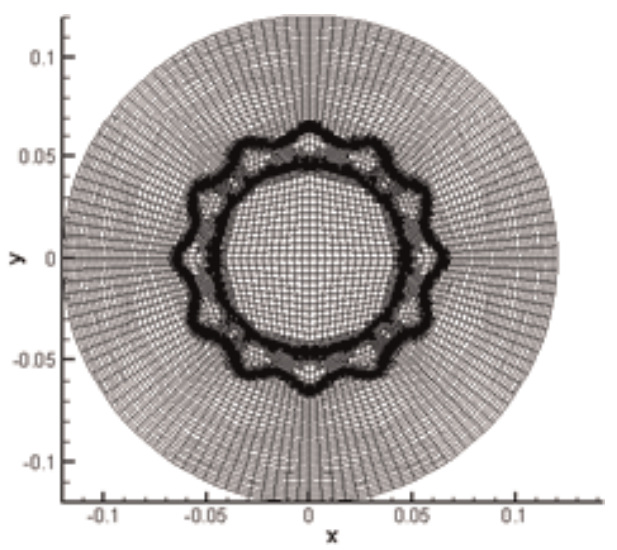

(a)

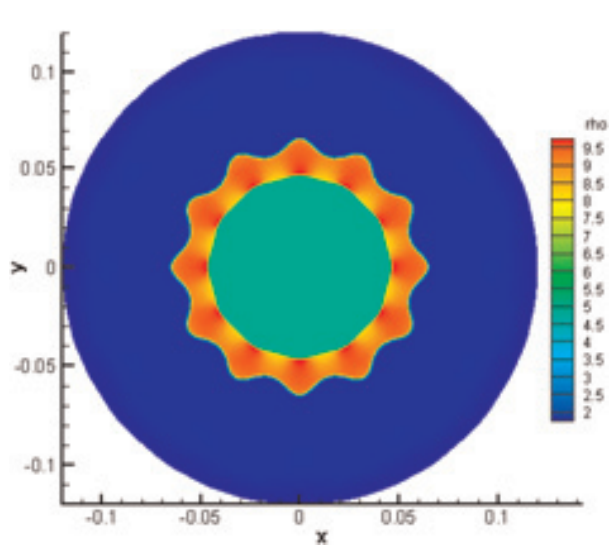

(b)

Fig. 12. (Color online) Mesh (a) and density contour (b) at time $t=0.15 \mathrm{~s}$. 


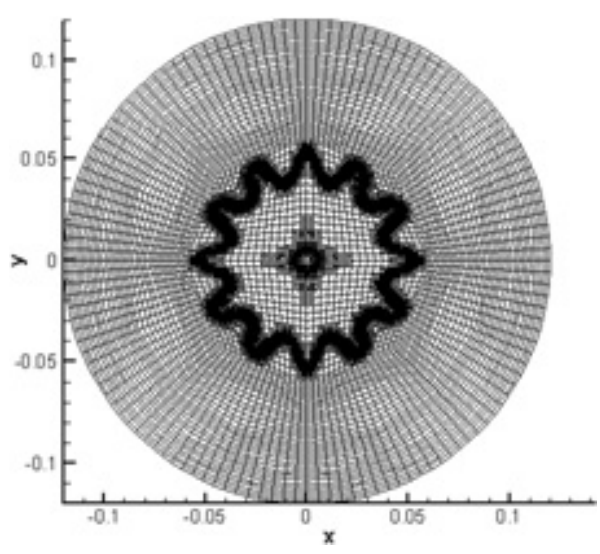

(a)

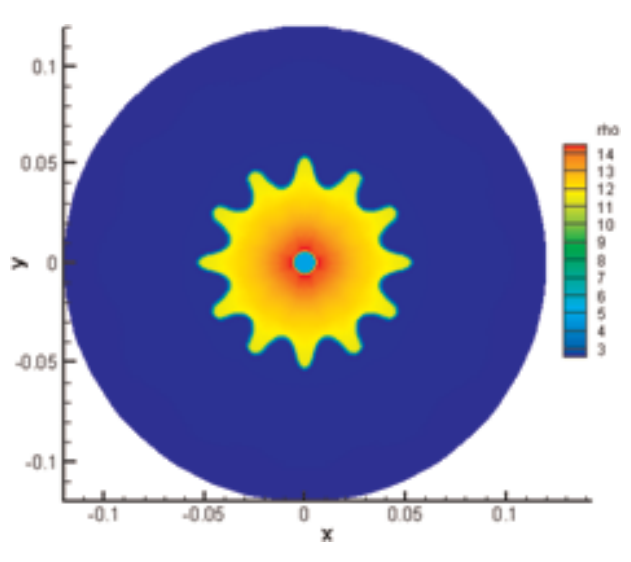

(b)

Fig. 13. (Color online) Mesh (a) and density contour (b) at time $t=0.35 \mathrm{~s}$.

and

$$
\rho_{\mathrm{SF} 6}=4.84 \mathrm{~kg} / \mathrm{m}^{3}, \quad \gamma_{\mathrm{SF} 6}=1.09, \quad \pi_{\mathrm{SF} 6}=0 .
$$

Similar to the first case, an imploding shock with Mach 1.2 is put at the circle with an initial radius of $7.5 \mathrm{~cm}$ to trigger the instability. These initial conditions are similar to the experiments, ${ }^{7}$ and the numerical investigation inside tube such as the front-tracking study ${ }^{11}$ and the PPM method. ${ }^{12}$

The simulation is performed on the adaptive mesh based on the background mesh (level is 0) with the finest resolution level as 4 . The results of mesh and density contour at different time from $0 \mathrm{~s}$ to $0.85 \mathrm{~ms}$ are plotted in Figs. 12-16. In early time (Fig. 12), the shock collides with the material interface and bifurcates into a

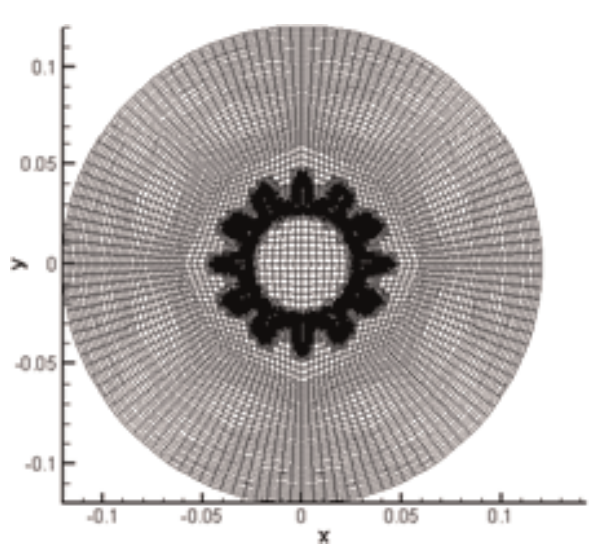

(a)

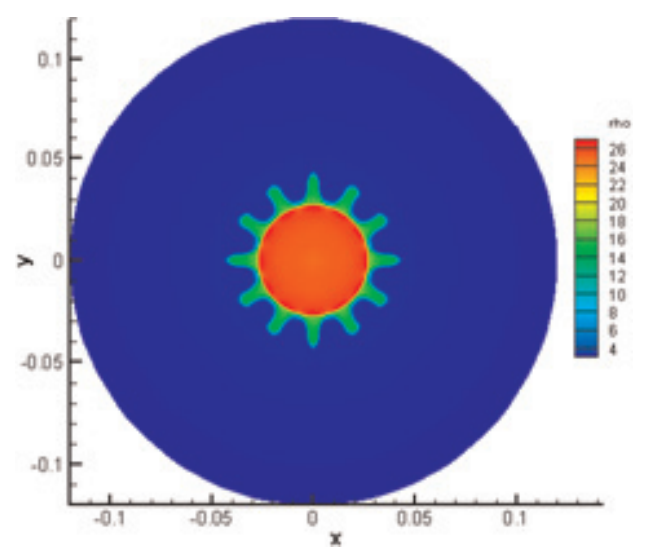

(b)

Fig. 14. (Color online) Mesh (a) and density contour (b) at time $t=0.55 \mathrm{~s}$. 


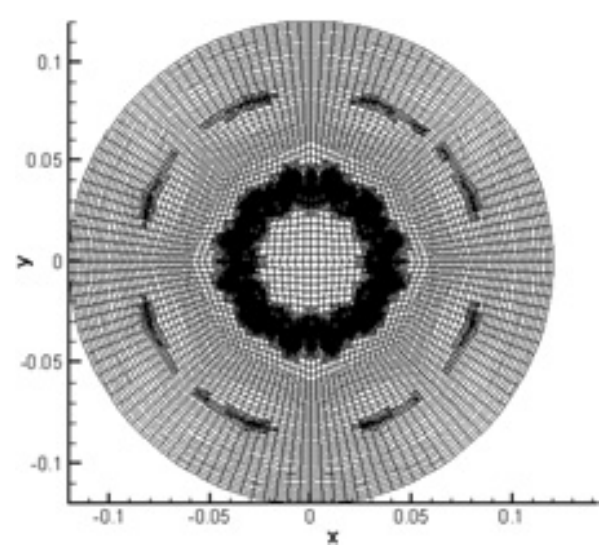

(a)

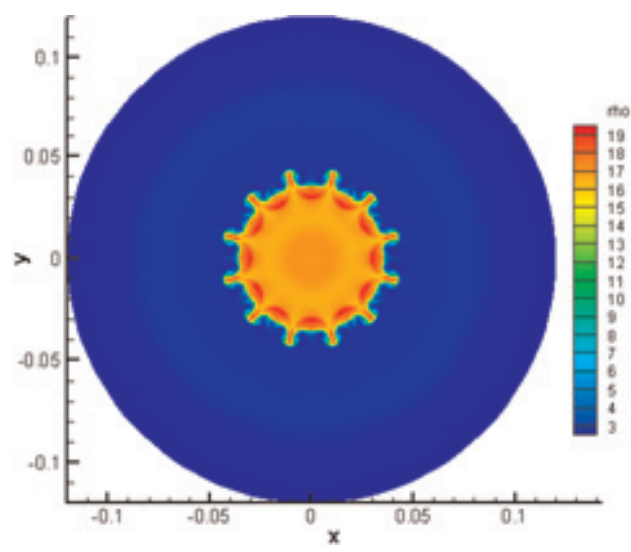

(b)

Fig. 15. (Color online) Mesh (a) and density contour (b) at time $t=0.7 \mathrm{~s}$.

transmitted shock moving toward the origin and a reflected shock traveling radically outward. It can be easily observed that the mesh are refined locally around the shock wave and interface. After that, the shock wave continues to move inward and is converging at the origin as shown in Fig. 13. It also drives the interface to be elongated (Fig. 13). The transmitted shock wave will reflect back from the origin moving outward and would drive the interface again, which is called re-shock (Fig. 14). The reflected shock from the origin will now travel in an opposite direction. It will hit the material interface and the interface has begun to roll up and formed the RM instability (Fig. 15). SF6 has greater density, so spikes are outward pointing fingers of heavy (SF6) fluid; bubbles are inward pointing fingers of air. The instability will

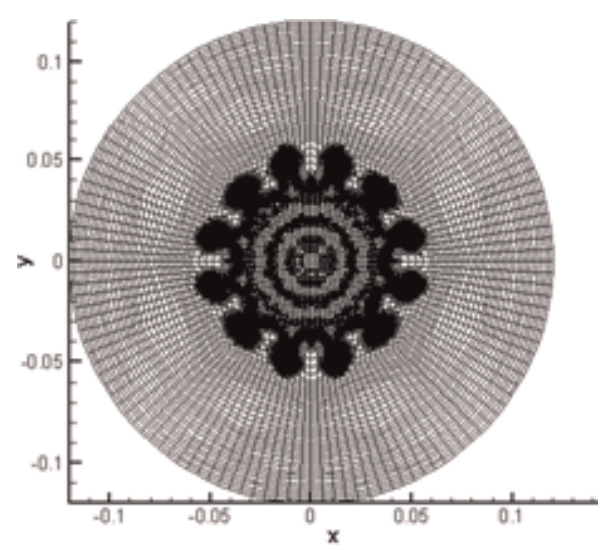

(a)

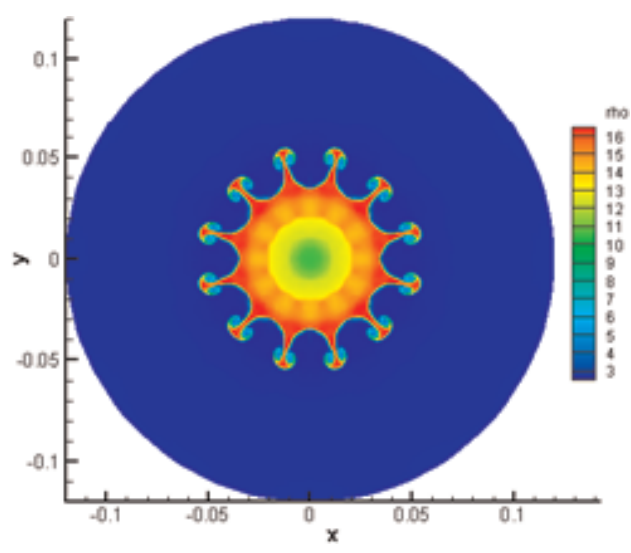

(b)

Fig. 16. (Color online) Mesh (a) and density contour (b) at time $t=0.85 \mathrm{~s}$. 
continue to develop to form the bubbles and spikes as the time goes. These could be clearly seen in Fig. 16.

\section{Conclusion}

In this paper, a solution adaptive method for compressible multi-fluid flows developed is applied to study the shock and interface interaction problem on a quadrilateral adaptive mesh. It is verified by the standard RM problem that the interfacial instability is induced by a planar shock. After that, the method is employed to investigate a circular shock interface interaction problem with relatively complex geometry. The results show the fine structures of the instability development due to shock reflection from the center (re-shock). These show the feasibility for us to study the compressible multi-fluid cases under complex geometry by the unstructured quadrilateral-based adaptive solver. ${ }^{15}$

\section{References}

1. S. W. Falk and W. D. Arnett, Astrophys. J. Lett. 180, 65 (1973).

2. W. D. Arnett, Astrophys. J. 319, 136 (1987).

3. W. D. Arnett, Astrophys. J. Suppl. Ser. 127, 123 (2000).

4. J. Kane, D. Arnett, B. A. Remington, S. G. Glendinning, G. Bazan, R. P. Drake, B. A. Fryxell, R. Teyssier and K. Moore, Phys. Plasmas 6, 2065 (1999).

5. J. D. Lindl, Phys. Plasmas 2, 3933 (1995).

6. M. Vetter and B. Sturtevant, Shock Waves 4, 247 (1995).

7. R. Benjamin, D. Besnard and J. Haas, Los Alamos National Laboratory Report No. LAUR 92-1185 (1993).

8. R. L. Holmes, J. W. Grove and D. H. Sharp, J. Fluid Mech. 301, 51 (1995).

9. K. M. Shyue, J. Comput. Phys. 142, 208 (1998).

10. R. R. Nourgaliev, T. N. Dinh and T. G. Theofanous, J. Comput. Phys. 213, 500 (2006).

11. Q. Zhang and M. J. Graham, Phys. Fluids 10, 974 (1998).

12. J. G. Zheng, T. S. Lee and S. H. Winoto, Math. Comput. Simul. 79, 749 (2008).

13. Y. Yang, Q. Zhang and D. H. Sharp, Phys. Fluids 6, 1856 (1994).

14. J. W. Banks, D. W. Schwendeman, A. K. Kapila and W. D. Henshaw, J. Comput. Phys. 223, 262 (2007).

15. H. W. Zheng, C. Shu and Y. T. Chew, J. Comput. Phys. 227, 6895 (2007).

16. R. Abgrall and S. Karni, J. Comput. Phys. 169, 594 (2001). 
Copyright of International Journal of Modern Physics C: Computational Physics \& Physical Computation is the property of World Scientific Publishing Company and its content may not be copied or emailed to multiple sites or posted to a listserv without the copyright holder's express written permission. However, users may print, download, or email articles for individual use. 